\title{
CORRIGENDUM
}

\section{Impact of nutrition counselling on nutrition knowledge and nutrient intake of 7- to 9-y-old children in an atherosclerosis prevention project}

\author{
M Räsänen et al \\ European Journal of Clinical Nutrition (2004) 58, 562. doi:10.1038/sj.ejcn.1601951
}

Correction to: European Journal of Clinical Nutrition (2004) 58, 162-172. doi:10.1038/sj.ejcn.1601763

Following the publication of the above paper, the authors have identified that one name was missing from the list of authors. Also, the affiliation was incorrect. The complete list of authors and their correct affiliations are reproduced below:

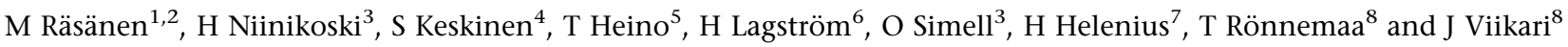

${ }^{1}$ The Research Centre of Applied and Preventive Cardiovascular Medicine, University of Turku, Finland; ${ }^{2}$ Tampere School of Public Health, University of Tampere, Finland; ${ }^{3}$ Department of Pediatrics, University of Turku, Finland; ${ }^{4}$ Department of Teacher Training in Rauma, University of Turku, Finland; ${ }^{5}$ The Research Centre of Applied and Preventive Cardiovascular Medicine, University of Turku, Finland; ${ }^{6}$ Departments of Biochemistry and Food Chemistry, University of Turku, Finland; ${ }^{7}$ Department of Biostatistics, University of Turku, Finland and ${ }^{8}$ Department of Medicine, University of Turku, Finland 